\title{
Third-harmonic generation in engineered slow light photonic crystal waveguides in chalcogenide glasses
}

\author{
Christelle Monat, ${ }^{1, *}$ Marcel Spurny, ${ }^{2}$ Christian Grillet, ${ }^{1}$ Liam O'Faolain, ${ }^{2}$ Thomas F. Krauss, ${ }^{2}$ \\ Benjamin J. Eggleton, ${ }^{1}$ Douglas Bulla, ${ }^{3}$ Steve Madden, ${ }^{3}$ and Barry Luther-Davies ${ }^{3}$ \\ ${ }^{1}$ CUDOS, Institute of Photonics and Optical Science (IPOS), School of Physics, University of Sydney, New South Wales 2006, Australia \\ ${ }^{2}$ School of Physics and Astronomy, University of St Andrews, St Andrews, Fife, KY16 9SS, UK \\ ${ }^{3}$ CUDOS, Laser Physics Centre, The Australian National University, Canberra, ACT 0200,Australia \\ Authore-mail address: christelle.monat@ec-lyon.fr
}

\begin{abstract}
We report third-harmonic generation in slow-light photonic crystal waveguides realized in chalcogenide glass membranes. This material enables a more uniform conversion along the waveguide and a higher efficiency than in comparable silicon structures.

OCIS codes: (130.5296) Photonic crystal waveguides; (190.4160) Multiharmonic generation
\end{abstract}

\section{Introduction}

Slow light propagation in photonic crystal $(\mathrm{PhC})$ waveguides has been shown to enhance the efficiency of nonlinear processes [1] including that of third-harmonic generation (THG) [2,3], making this platform promising for realizing short nonlinear devices with low power consumption. The possibility of engineering the dispersion in these structures makes it possible to achieve nonlinear functions that can operate at high bit rates, with a record of $640 \mathrm{Gbit} / \mathrm{s}$ reported [4]. However, most of these demonstrations use silicon or III-V materials with a nonlinear figure of merit FOM $\mathrm{n}_{2} /$ TPA $\left(\mathrm{n}_{2}\right.$ and TPA are the optical Kerr and two-photon absorption (TPA) coefficients), lower than 1 , which is undesirable for the device performance. Some groups have started exploring materials with a larger FOM such as wide band gap III-Vs [5,6] and chalcogenide glasses [7-9], but the associated demonstrations still rely on band-edge slow light where loss and dispersion are typically high. Besides, the fabrication quality in such less mature materials remains challenging, resulting in $\mathrm{PhC}$ waveguides with typically larger propagation losses ( $\sim 170 \mathrm{~dB} / \mathrm{cm}$ for $\mathrm{c} / 5$ modes in $\mathrm{Ag}$-doped chalcogenide $\mathrm{PhC}$ waveguides [9]).

Here, we report on the generation of visible light from the conversion of a near-infrared (NIR) signal through THG in a slow light $\mathrm{PhC}$ waveguide fabricated in a chalcogenide glass. We achieve both reasonably low propagation losses and engineered dispersion with a "flat-band" slow light window (group velocity $\mathrm{v}_{\mathrm{g}} \sim \mathrm{c} / 30$ ). Combined with the low nonlinear loss of this material (FOM estimated to 5-10 for bulk [7]), this allows us to demonstrate THG with a higher conversion efficiency than in silicon [2]. The pattern of visible light generated along the waveguide is also more uniform, which widens the field of applications of THG in PhC waveguides.

2. Slow light dispersion engineered $\mathrm{PhC}$ waveguides in chalcogenide
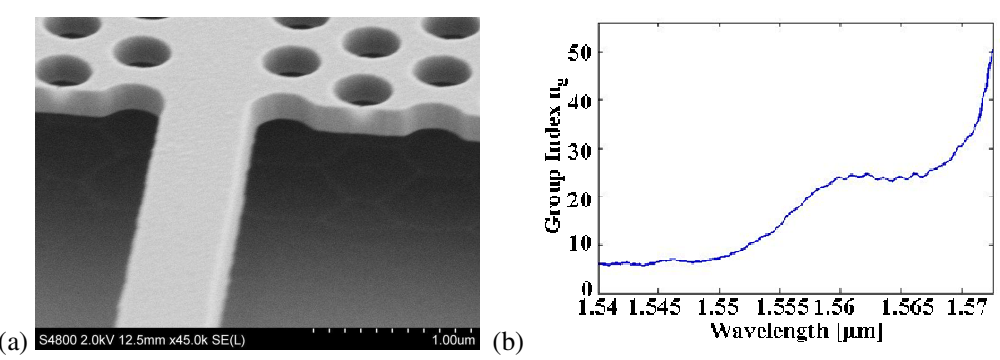

Fig. 1. (a) SEM picture of a chalcogenide PhC waveguide including the tapered access nanowire and (b) Measured group index dispersion.

Our sample consists of a 100 m long AMTIR-1 (Ge33As12Se55) chalcogenide PhC waveguide suspended in air and connected on both sides to tapered ridge waveguides. The chip was fabricated using e-beam lithography, chemically assisted ion beam etching and vapour phase HF etching on a 300nm thick AMTIR-1 film (measured index $\mathrm{n} \sim 2.6$ [10]) evaporated onto a $\mathrm{SiO}_{2} / \mathrm{Si}$ wafer. The $\mathrm{PhC}$ waveguide was created by omitting one row of holes in the $\mathrm{K}$ direction of the triangular $\mathrm{PhC}$ lattice (period $\mathrm{a} \sim 525 \mathrm{~nm}$ and hole radius $\sim 0.3 \mathrm{a}$ ), and the first two rows of holes around the defect were laterally shifted to engineer the dispersion of the fundamental mode [11]. A short (five periods) $\mathrm{PhC}$ adapter (stretched a 555nm along the waveguide axis) was inserted at both ends of the PhC waveguide to improve light insertion into the slow light mode. Figure $1 \mathrm{~b}$ shows the measured group index dispersion that exhibits a nearly constant $\mathrm{v}_{\mathrm{g}}$ of $\sim \mathrm{c} / 305 \%$ over a $\sim 10 \mathrm{~nm}$ bandwidth. 


\section{Experimental results}

We probe the waveguide in the nonlinear regime using lensed fibers to butt-couple the train of $1.2 \mathrm{ps}$ pulses $(4 \mathrm{MHz}$ repetition rate) arising from a mode-locked fiber laser at a wavelength tunable around 1550nm. The NIR pump signal is amplified and TE-polarized before coupling into the chip with an efficiency of $\sim 14 \%$. Fig. 2a shows the transmission of the NIR signal when increasing the coupled power up to $\sim 5 \mathrm{~W}$ peak (200 W input average). No significant saturation of the transmission, as a signature of nonlinear loss, is observed in this power range.
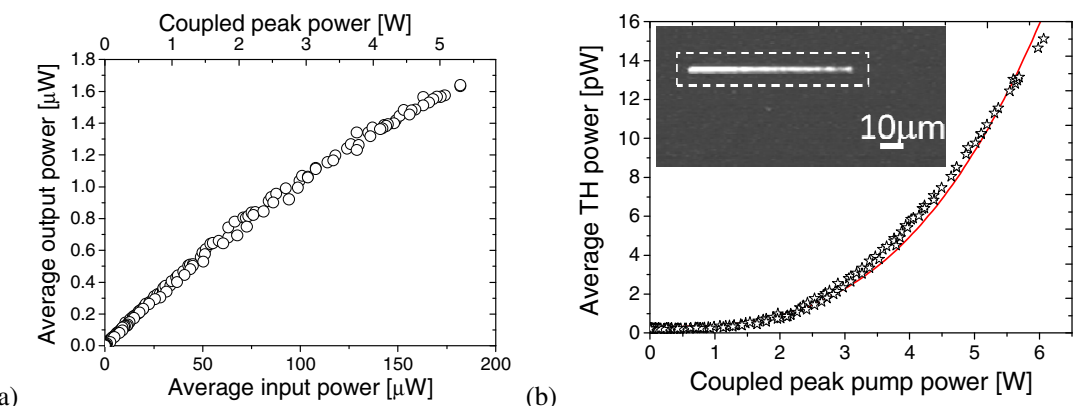

Fig. 2. (a) NIR pump transmission. (b) Average power of visible TH light versus coupled peak pump power along with a cubic fit. The inset is a CCD image of the TH light generated at $\sim 520 \mathrm{~nm}$.

A fraction of the NIR pump is converted into visible third-harmonic (TH) light. The inset of Fig. 2b shows a CCD image of the TH light collected out-of-plane through a N.A. $=0.42$ objective. Note that the pattern of visible light is relatively uniform along the waveguide length. This in contrast with what had been observed in $\mathrm{Si} \mathrm{PhC}$ waveguides, where the TH light was rapidly decaying over a short distance of propagation [2]. This originates from the lower TPA induced nonlinear losses and the absence of free carriers generated in chalcogenide. Figure $2 b$ shows the measured average power of TH visible light $\left(\mathrm{P}_{3}\right)$ as a function of the pump power $(\mathrm{P})$ along with a cubic fit -characteristic of the THG process $\left(\mathrm{P}_{3} \quad \mathrm{P}^{3}\right)$. No major deviation from the cubic fit is observed, reemphasizing the negligible contribution of nonlinear loss in this material for the power considered. The inferred THG conversion efficiency $\mathrm{P}_{3} / \mathrm{P}$ is found equal to $\sim 1,4 \times 10^{-8}$ for $1 \mathrm{~W}$ coupled peak power, which is $\sim 30 \times$ larger than what was measured in comparable silicon slow light $\mathrm{PhC}$ waveguides [2]. Like for $\mathrm{Si}$, this represents a lower bound for the conversion efficiency, due to the limited extraction of the TH light from a material opaque in the visible.

In conclusion, we have demonstrated THG in a slow light dispersion engineered $\mathrm{PhC}$ waveguide made of chalcogenide. Due to their relatively high nonlinear figure of merit, chalcogenide glasses provide a suitable platform for exploiting slow light enhanced nonlinear processes, and compositions with even larger FOM are available and could be advantageously used. Besides an increase in the THG conversion efficiency, these better material properties translate into a more uniform pattern of visible light generated along the waveguide, which opens up new opportunities for applying THG to the temporal measurement of ultra-fast optical signals using on-chip devices.

\section{References}

[1] C. Monat, B. Corcoran, D. Pudo, M. Ebnali-Heidari, C. Grillet, M. D. Pelusi, D. J. Moss, B. J. Eggleton, T. P. White, L. O’Faolain, and T. F. Krauss, "Slow light enhanced nonlinear optics in silicon photonic crystal waveguides," J. Sel. Top. Quantum Electron 16, 344-356 (2010).

[2] B. Corcoran, C. Monat, C. Grillet, D. J. Moss, B. J. Eggleton, T. P. White, L. O'Faolain, and T. F. Krauss, "Green light emission in silicon through slow-light enhanced third-harmonic generation in photonic-crystal waveguides," Nat. Photonics 3, 206-210 (2009).

[3] C. Monat, C. Grillet, B. Corcoran, D. J. Moss, B. J. Eggleton, T. P. White, and T. F. Krauss, "Investigation of phase matching for thirdharmonic generation in silicon slow light photonic crystal waveguides using Fourier optics," Opt. Express 18, 6831-6840 (2010).

[4] B. Corcoran, C. Monat, M.Pelusi, C. Grillet, T. P. White, L. O'Faolain, T. F. Krauss, B. J. Eggleton and D. J. Moss, "Optical signal processing on a silicon chip at 640Gb/s using slow-light," Opt. Express 18, 7770-7781 (2010).

[5] S. Combrie, Q. V. Tran, A. De Rossi, C. Husko, and P. Colman, "High quality GaInP nonlinear photonic crystals with minimized nonlinear absorption," Appl. Phys. Lett. 95, 221108 (2009).

[6] K. Rivoire, Z. Lin, F. Hatami, W. Ted Masselink, and J. Vuckovic, "Second harmonic generation in gallium phosphide photonic crystal nanocavities with ultralow continuous wave pump power", Opt. Express 17, 22609-22615 (2009).

[7] Y. Ruan, M.-K. Kim, Y.-H. Lee, B. Luther-Davies and A. Rode, "Fabrication of high-Q chalcogenide photonic crystal resonators by e-beam lithography", Appl. Phys. Lett. 90, 071102 (2007).

[8] M. W. Lee, C. Grillet, C. G. Poulton, C. Monat, C. L. C. Smith, E. Mägi, D. Freeman, S. Madden, B. Luther-Davies, and B. J. Eggleton, "Characterizing photonic crystal waveguides with an expanded k-space evanescent coupling technique," Opt. Express 16, 13800-08 (2008).

[9] K. Suzuki, Y. Hamachi, and T. Baba, "Fabrication and characterization of chalcogenide glass photonic crystal waveguides," Opt. Express 17, 22393-22400 (2009).

[10]M.Spurny, L. O'Faolain, D.A.P Bulla, B. Luther-Davies and T.F. Krauss, "Fabrication of low loss dispersion engineered chalcogenide photonic crystals", submitted to Opt. Express.

[11]S. A. Schulz et al. "Dispersion Free Slow Light in Photonic Crystals: A Comparison,” Journal of Optics 12, 104004 (2010). 